\title{
Reevaluation of the Phylogenetic Depth of the Marine Aerobic Hyperthermophilic Archaeon Aeropyrum pernix Based on Comparative Analysis of 23S rRNA Sequences
}

\author{
Norimichi Nomura, Yoshihiko Sako, and Aritsune Uchida \\ Laboratory of Marine Microbiology, Division of Applied Bioscience, Graduate School of Agriculture, \\ Kyoto University, Kyoto 606-8502, Japan
}

(Received August 21, 1998)

\begin{abstract}
Aeropyrum pernix, a strictly aerobic marine hyperthermophilic archaeon, has been studied as a potential biotechnological source. In order to clarify the phylogenetic position of $A$. pernix, the nearly complete nucleotide sequence of the 23S rRNA was determined and compared with the other hitherto known archaeal counterparts. To avoid the artifact caused by disparities of $\mathrm{G}+\mathrm{C}$ content, transversion evolutionary distance analysis was adopted. The result confirmed that $A$. pernix is closely allied to the Pyrodictium-Desulfurococcus-Staphylothermus group (the order "Igneococcales") within the kingdom Crenarchaeota. However, it also revealed that the genus Aeropyrum diverges sharply from the other genera within the family Desulfurococcaceae. Thus, we concluded that the decision to transfer the genus Aeropyrum from the family Desulfurococcaceae is reasonable in light of our present data, as well as the physiological characteristics of this archaeon reported previously.
\end{abstract}

Key words: Aeropyrum pernix, molecular phylogeny, archaea, Crenarchaeota, 23S rRNA, hyperthermophile, transversion evolutionary distance

Marine hyperthermophilic archaea (formerly called archaebacteria) have potential biotechnological and commercial significance. They have provided a lot of valuable resources for exploitation in novel industrial processes and in developing our understanding of how biomolecules are stabilized when under extremely high temperatures. Over the past fifteen years, a number of novel archaea have been isolated from marine hydrothermal environments, such as submarine hot springs and vents. ${ }^{1,2)} \mathrm{We}$ have also isolated a novel archaeon Aeropyrum pernix at a coastal solfataric vent in Kodakara Island, Japan. ${ }^{3,4)} \boldsymbol{A}$, pernix is a strictly aerobic heterotroph growing optimally at 90 to $95^{\circ} \mathrm{C}$ and may therefore be well suited for biotechnological application of thermostable enzymes. Various types of proteinases are being developed for this purpose, ${ }^{5,6)}$ and genes for rRNA intron-encoded homing endonucleases ${ }^{7, *}$ and DNA polymerase (Ishino et al., Personal communication) have been isolated from this organism.

Our previous study suggested that $A$. pernix is a distinctive lineage related to the genera Pyrodictium and Desulfurococcus within the kingdom Crenarchaeota. ${ }^{3)}$ However, the valid phylogenetic position of $A$. pernix was difficult to determine, since reconstitution of the neighborjoining phylogenetic tree based on 16S rRNA sequences were ambiguous. Moreover, only a limited number of sequences were used for the analysis due to variation of the biased base composition. Subsequently, Burggraf et al. reported that the most probable 16S rRNA-based phylogenetic trees determined by most algorithms place the genus Aeropyrum in the family Desulfurococcaceae, although occasionally some algorithms place this genus in the family Pyrdictiaceae. ${ }^{8)}$ These results suggest that genetic information of 16S rRNA is not enough to determine the exact and robust position of $A$. pernix.

23S rRNA shares the features as a molecular clock with 16S rRNA and has the added advantages of an even longer chain length, which might provide more reliable information for inferring phylogenies. ${ }^{9}$ Therefore, we determined the nearly complete 23S rRNA sequence of $A$. pernix and resolved the phylogeny of the genus Aeropyrum.

\section{Materials and Methods}

\section{Cultivation, Preparation of $23 S r R N A$, and Sequencing}

Aeropyrum pernix (strain K1; JCM 9820) cells were grown in JXT medium at $90^{\circ} \mathrm{C}$ with vigorous shaking and harvested in the late exponential phase. ${ }^{3)} 23 \mathrm{~S}$ rRNA was extracted and purified as previously reported. ${ }^{7}$ The cDNA clone of the 23S rRNA was obtained after reverse transcription and PCR amplification. The oligonucleotide (DmLR3070R, 5'-CGGGCTCTTGGGAGCGGCG-3'), complementary to the $3^{\prime}$-tail region of the 23S rRNA of Desulfurococcus mobilis ${ }^{10)}$ was used to prime first-strand synthesis with Superscript II RNaseH- (GIBCO Laboratories, Grand Island, N.Y.). The reverse transcription reaction was performed according to the manufacture's instructions, and the product was amplified by PCR with 0227aF (5'-GAGGAAAAGAAATCAA-3 $\left.3^{\prime}\right)^{11}$ and DmLR3070R as primers. Thirty five amplification cycles of $90 \mathrm{~s}$ at $96^{\circ} \mathrm{C}, 1 \mathrm{~min}$ at $62^{\circ} \mathrm{C}$, two minutes at $72^{\circ} \mathrm{C}$ were

* N. Nomura, Y. Sako, Y. Morinaga, and A. Uchida: Two latent homing endonucleases encoded in the rRNA introns of the hyperthermophilic archaeon Aeropyrum pernix K1. Abst. of the International Congress on Extremophiles '98, January, 1998, p. 208. 
performed. The double-stranded PCR product was cloned directly into the pCRII vector (Invitrogen, San Diego, Ca.) to yield plasmid pNB23. Deletion mutants were produced by progressive exonuclease III digestion, ${ }^{12)}$ and the nucleotide sequences were determined by the dideoxy sequencing method. ${ }^{13)}$

\section{Alignment and Phylogenetic Methods}

The $A$. pernix $23 \mathrm{~S}$ rRNA sequence was aligned using the CLUSTAL $W{ }^{14)}$ to the sequences previously described for 24 Archaea and 1 Bacterium available from the DDBJ, EMBL, and GenBank nucleotide sequence databases and from LSU rRNA database ${ }^{15)}$ and checked manually. Nucleotides undetermined for any of the sequences and regions of uncertain alignment were omitted from the analysis and evolutionary distances were calculated for 1,242 nucleotides from position 1,261 to $2,788(E$. coli numbering system ${ }^{16}$ ) using the correction of Kimura's 2parameter model. ${ }^{17)}$ The phylogenetic tree was constructed by the neighbor-joining method ${ }^{18)}$ from the transversion evolutionary distance matrix. ${ }^{19)}$ Bootstrap analysis ${ }^{20)}$ was used to generate confidence estimates for tree topologies.

\section{Nucleotide Sequence Accession Number}

The 23S rRNA sequence of $A$. pernix will appear in the DDBJ, EMBL, and GenBank nucleotide sequence databases with the accession number AB004787.

\section{Results}

The partial 23S rRNA sequence of $A$. pernix, from positions 228 to 2871 (according to $E$. coli numbering) representing 2842 nucleotides, was determined. The $\mathrm{G}+\mathrm{C}$ content of this sequence was $69.0 \mathrm{~mol} \%$.

The sequences employed in the comparative analysis had $\mathrm{G}+\mathrm{C}$ contents in the range of 51.8 to $67.6 \mathrm{~mol} \%$, while purine contents ranged from 55.1 to $56.6 \mathrm{~mol} \%$. Therefore, to avoid the artifact caused by compositional disparities, transversion evolutionary distances were estimated (Table 1), and a phylogenetic tree was constructed by the neighbor-joining method (Fig. 1). Approximate topology of the tree was similar to the reported one based on $16 \mathrm{~S}$ rRNA ${ }^{21)}$ with the exception of the convergence between the order Thermococcales and the cluster of the orders Methanococcales-Methanobacteriales. According to the phylogenetic tree, the kingdom Crenarchaeota is composed of three major branches corresponding to three orders, Thermoproteales, Sulfolobales, "Igneococcales". The tree showed that $A$. pernix was a deeply branching representative, which was located above the order Sulfolobales and below branching the family Pyrodictiaceae from the family Desulfurococcaceae within the kingdom Crenarchaeota. The bootstrap value of $100 \%$ among 1000 resampling supported the grouping of the genus Aeropyrum, the family Pyrodictiaceae, and the family Desulfurococcaceae. Besides, the bootstrap value for the nodes branching the three species Pyrodictium occultum, Desulfurococcus mobilis, and Staphylothermus marinus were relatively high ( $85 \%$ and $87 \%$ ), indicating the probability of their branching order. Particularly noteworthy was that $A$. pernix represented the second shortest, therefore primitive, lineage in this archaeal tree.

$A$. pernix $23 \mathrm{~S}$ rRNA shared a secondary structural feature with the families Pyrodictiaceae and Desulfurococcaceae that might be a defining signature of this particular group (Fig. 2). The helix at positions 1445 to 1465 in the 23S rRNA secondary structure ( $E$. coli numbering) of all

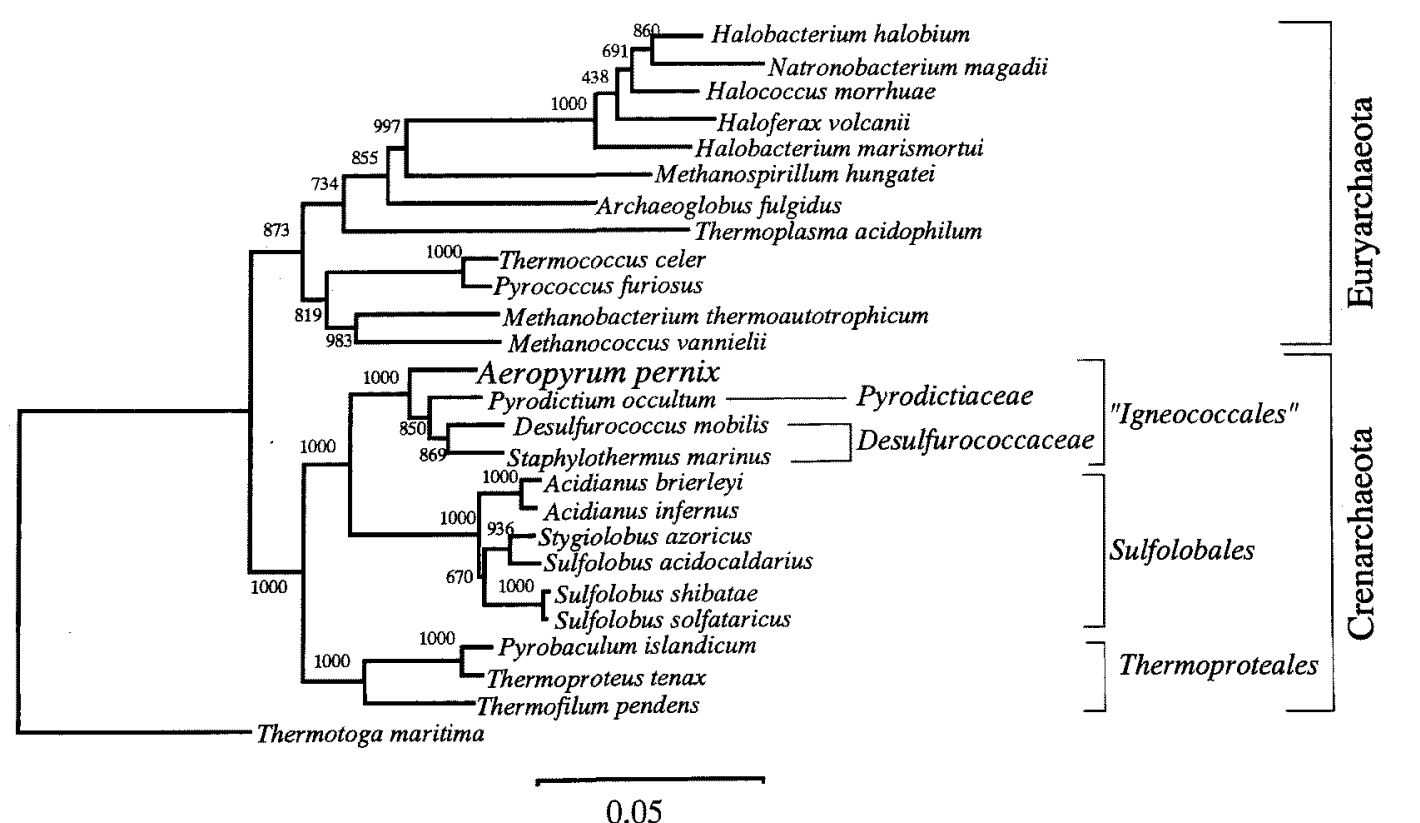

\subsection{5}

Fig. 1. Phylogenetic relationships for the crenarchaea and the position of Aeropyrum pernix with 23S rRNA transversion evolutionary distances of Table 1.

Bar represents 5 transversional nucleotide substitution per 100 site. The sequence of the bacterium Thermotoga maritima was used as an outgroup. Numbers at nodes represent bootstrap values for that node based on 1000 bootstrap resamplings. 
Table 1. Nucleotide ratios and transversion evolutionary distances among representative 25 archaeal and 1 bacterial $23 \mathrm{~S}$ rRNA sequences

\begin{tabular}{|c|c|c|c|c|c|c|c|c|c|c|c|c|c|c|c|c|c|c|c|c|c|c|c|c|c|c|c|c|}
\hline Species & $\begin{array}{l}G+C \\
\text { mol } \%\end{array}$ & $\begin{array}{l}A+G \\
\mathrm{~mol} \%\end{array}$ & 1 & 2 & 3 & 4 & 5 & 6 & 7 & 8 & 9 & 10 & 11 & 12 & 13 & 14 & 15 & 16 & 17 & 18 & 19 & 20 & 21 & 22 & 23 & 24 & 25 & 26 \\
\hline 1 Aeropyrum pernix & 67.6 & 55.6 & - & & & & & & & & & & & & & & & & & & & & & & & & & \\
\hline 2 Pyrodictium occultum & 67.1 & 55.9 & 0.0314 & - & & & & & & & & & & & & & & & & & & & & & & & & \\
\hline 3 Desulfurococcus mobilis & 65.6 & 55.5 & 0.0338 & 0.0314 & - & & & & & & & & & & & & & & & & & & & & & & & \\
\hline 4 Staphylothermus marinus & 66.3 & 56.2 & 0.0346 & 0.0242 & 0.0250 & - & & & & & & & & & & & & & & & & & & & & & & \\
\hline 5 Acidianus brierleyi & 61.9 & 56.2 & 0.0701 & 0.0741 & 0.0733 & 0.0709 & - & & & & & & & & & & & & & & & & & & & & & \\
\hline 6 Acidianus infernus & 63.0 & 56.3 & 0.0693 & 0.0749 & 0.0693 & 0.0701 & 0.0073 & - & & & & & & & & & & & & & & & & & & & & \\
\hline 7 Sulfolobus acidocaldarius & 62.4 & 56.1 & 0.0725 & 0.0701 & 0.0741 & 0.0733 & 0.0266 & 0.0258 & - & & & & & & & & & & & & & & & & & & & \\
\hline 8 Sulfolobus shibatae & 63.0 & 56.2 & 0.0766 & 0.0693 & 0.0717 & 0.0725 & 0.0274 & 0.0282 & 0.0266 & - & & & & & & & & & & & & & & & & & & \\
\hline 9 Sulfolobus solfataricus & 63.0 & 56.3 & 0.0757 & 0.0685 & 0.0709 & 0.0717 & 0.0282 & 0.0290 & 0.0274 & 0.0024 & - & & & & & & & & & & & & & & & & & \\
\hline 10 Stygiolobus azoricus & 62.9 & 56.2 & 0.0717 & 0.0693 & 0.0717 & 0.0709 & 0.0258 & 0.0234 & 0.0121 & 0.0242 & 0.0250 & - & & & & & & & & & & & & & & & & \\
\hline 11 Thermofitum pendens & 65.0 & 56.4 & 0.0774 & 0.0766 & 0.0790 & 0.0798 & 0.0878 & 0.0870 & 0.0919 & 0.0975 & 0.0967 & 0.0878 & - & & & & & & & & & & & & & & & \\
\hline 12 Thermoproteus tenax & 65.1 & 55.9 & 0.0717 & 0.0725 & 0.0749 & 0.0806 & 0.0951 & 0.0959 & 0.0975 & 0.0951 & 0.0943 & 0.0935 & 0.0508 & - & & & & & & & & & & & & & & \\
\hline 13 Pyrobaculum islandicum & 65.9 & 56.3 & 0.0709 & 0.0733 & 0.0790 & 0.0798 & 0.0991 & 0.0983 & 0.0999 & 0.0975 & 0.0967 & 0.0959 & 0.0516 & 0.0105 & - & & & & & & & & & & & & & \\
\hline 14 Thermococcus celer & 63.3 & 55.9 & 0.0959 & 0.0983 & 0.1023 & 0.1080 & 0.1209 & 0.1185 & 0.1217 & 0.1225 & 0.1217 & 0.1209 & 0.0991 & 0.0951 & 0.0975 & - & & & & & & & & & & & & \\
\hline 15 Pyrococcus furiosus & 64.3 & 55.8 & 0.0870 & 0.0943 & 0.0967 & 0.1039 & 0.1201 & 0.1176 & 0.1209 & 0.1249 & 0.1241 & 0.1185 & 0.1015 & 0.0959 & 0.0983 & 0.0137 & - & & & & & & & & & & & \\
\hline 16 Methanococcus vannielii & 57.2 & 55.9 & 0.1056 & 0.1048 & 0.1152 & 0.1144 & 0.1176 & 0.1201 & 0.1185 & 0.1257 & 0.1249 & 0.1209 & 0.1023 & 0.1064 & 0.1120 & 0.0774 & 0.0733 & - & & & & & & & & & & \\
\hline $\begin{array}{l}17 \text { Methanobacterium } \\
\text { thermoautotrophicum }\end{array}$ & 58.1 & 55.4 & 0.0959 & 0.1064 & 0.1088 & 0.1160 & 0.1176 & 0.1168 & 0.1135 & 0.1176 & 0.1168 & 0.1144 & 0.1039 & 0.1015 & 0.1039 & 0.0757 & 0.0749 & 0.0629 & - & & & & & & & & & \\
\hline $\begin{array}{c}18 \text { Thermoplasma } \\
\text { acidophilum }\end{array}$ & 54.9 & 55.5 & 0.1571 & 0.1483 & 0.1620 & 0.1612 & 0.1628 & 0.1636 & 0.1555 & 0.1579 & 0.1571 & 0.1547 & 0.1475 & 0.1467 & 0.1475 & 0.1273 & 0.1297 & 0.1418 & 0.1305 & - & & & & & & & & \\
\hline 19 Archaeoglobus fulgidus & 62.2 & 56.6 & 0.1305 & 0.1330 & 0.1370 & 0.1330 & 0.1346 & 0.1354 & 0.1338 & 0.1378 & 0.1370 & 0.1346 & 0.1338 & 0.1378 & 0.1386 & 0.1023 & 0.0999 & 0.0975 & 0.1104 & 0.1313 & - & & & & & & & \\
\hline $\begin{array}{l}20 \text { Methanospirillum } \\
\text { hungatei }\end{array}$ & 51.8 & 55.9 & 0.1426 & 0.1402 & 0.1475 & 0.1434 & 0.1547 & 0.1555 & 0.1539 & 0.1563 & 0.1555 & 0.1547 & 0.1426 & 0.1467 & 0.1523 & 0.1225 & 0.1233 & 0.1176 & 0.1241 & 0.1467 & 0.1039 & - & & & & & & \\
\hline $\begin{array}{l}\text { 21 Halobacterium } \\
\text { marismortui }\end{array}$ & 56.2 & 55.5 & 0.1459 & 0.1515 & 0.1555 & 0.1547 & 0.1595 & 0.1571 & 0.1620 & 0.1676 & 0.1668 & 0.1595 & 0.1394 & 0.1483 & 0.1491 & 0.1338 & 0.1346 & 0.1273 & 0.1241 & 0.1515 & 0.1168 & 0.1193 & - & & & & & \\
\hline 22 Haloferar valcanit & 55.7 & 55.4 & 0.1516 & 0.1589 & 0.1597 & 0.1589 & 0.1669 & 0.1645 & 0.1677 & 0.1734 & 0.1726 & 0.1669 & 0.1468 & 0.1540 & 0.1548 & 0.1411 & 0.1419 & 0.1250 & 0.1202 & 0.1637 & 0.1177 & 0.1234 & 0.0556 & - & & & & \\
\hline 23 Halococcus morrhuae & 58.0 & 54.7 & 0.1483 & 0.1523 & 0.1579 & 0.1555 & 0.1636 & 0.1612 & 0.1644 & 0.1716 & 0.1708 & 0.1636 & 0.1418 & 0.1507 & 0.1515 & 0.1378 & 0.1386 & 0.1281 & 0.1265 & 0.1571 & 0.1144 & 0.1185 & 0.0363 & 0.0419 & - & & & \\
\hline $\begin{array}{l}24 \text { Natronobacterium } \\
\text { magadii }\end{array}$ & 56.8 & 55.1 & 0.1660 & 0.1700 & 0.1757 & 0.1732 & 0.1829 & 0.1805 & 0.1869 & 0.1894 & 0.1886 & 0.1845 & 0.1612 & 0.1668 & 0.1676 & 0.1491 & 0.1499 & 0.1442 & 0.1362 & 0.1636 & 0.1241 & 0.1249 & 0.0459 & 0.0452 & 0.0403 & - & & \\
\hline 25 Halobacterium halobium & 55.7 & 55.7 & 0.1539 & 0.1563 & 0.1652 & 0.1595 & 0.1644 & 0.1620 & 0.1652 & 0.1708 & 0.1700 & 0.1676 & 0.1410 & 0.1531 & 0.1523 & 0.1370 & 0.1378 & 0.1289 & 0.1176 & 0.1531 & 0.1072 & 0.1160 & 0.0403 & 0.0460 & 0.0346 & 0.0363 & - & \\
\hline 26 Thermotoga maritima & 62.7 & 56.6 & 0.1531 & 0.1555 & 0.1579 & 0.1587 & 0.1652 & 0.1644 & 0.1692 & 0.1716 & 0.1692 & 0.1684 & 0.1483 & 0.1555 & 0.1547 & 0.1523 & 0.1531 & 0.1684 & 0.1620 & 0.1910 & 0.1902 & 0.1797 & 0.2006 & 0.1984 & 0.1966 & 0.2111 & 0.1974 & - \\
\hline
\end{tabular}

For nucleotide ratios, only positions used in the phylogenetic analysis were included in this calculation.

The sequences used in this calculations were obtained from DDBJ/EMBL/GenBank nucleotide sequence databases with the exception of the sequence of Haloferax volcanii and Thermoproteus tenax, which were extracted from LSU The sequences used in this calculations were obtained from DDBJ/EMBL/GenBank nucleotide sequence databases with the exception of the sequence of Haloferax volcanii and Thermoproteus tenax, which were extracted from LSU
rRNA database. ${ }^{15}$ Nucleotide sequence accession numbers are as follows: Pyrodictium occultum, M86626; Desulfurococcus mobilis, X05480; Staphylothermus marinus, M86623; Acidianus brierleyi, U32317; Acidianus infernus, U32318;

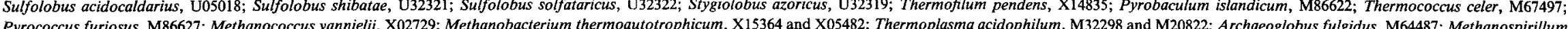
Pyrococcus furiosus, M827, M. total of 1242 unambiguous nucleotides were used in the analysis. 


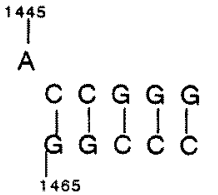

Aeropyrum pernix

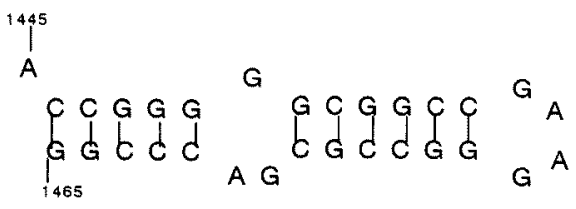

Pyrodictium occultum

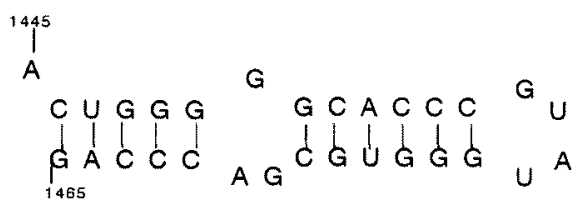

Desulfurococcus mobilis

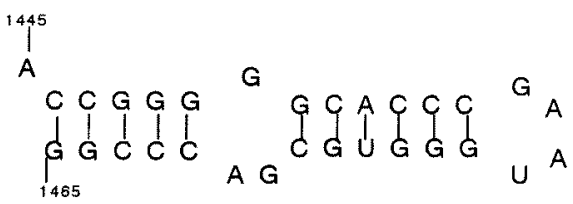

Staphylothermus marinus

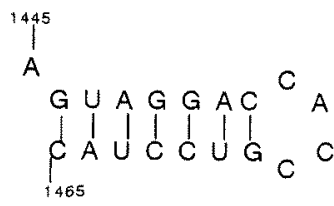

Sulfolobus acidocaldarius

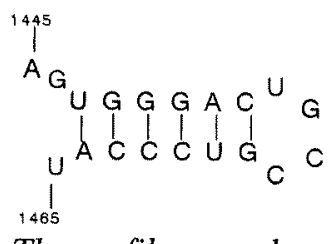

Thermofilum pendens

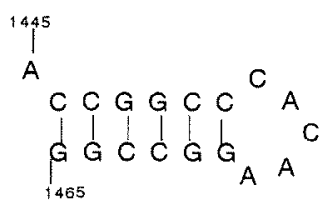

Thermococcus celer

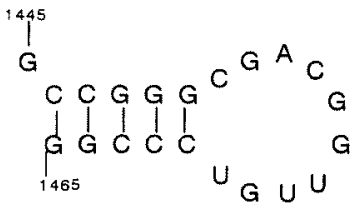

Escherichia coli

Fig. 2. Diagnostic putative secondary structure of the 1445-to-1465 region (E. coli numbering) of several 23S rRNAs which distinguishes the members of the order "Igneococcales" from the other archaea.

sequences was characterized by a $G$ bulge 6 nucleotides $5^{\prime}$ from the capping loop and a AG bulge 6 nucleotides $3^{\prime}$ from the capping loop. This motif was not found elsewhere in the domain Archaea.

\section{Discussion}

Burggraf et al. described that the genus Aeropyrum could be assigned to the family Desulfurococcaceae in accord to the 16S rRNA-based phylogenetic analyses. ${ }^{8)}$ However, the tree topology was considerably unstable, therefore, the above conclusion is not necessarily valid from a statistical point of view. Therefore, we reexamined the crenarchaeal phylogeny on the basis of $23 \mathrm{~S}$ rRNA sequences and determined the relationship of the genus Aeropyrum to the genera in the family Desulfurococcaceae with whom it is currently grouped.

Due to the different base substitution rates of evolution in 23S rRNA sequences employed in this analysis (Table. 1), it is difficult to define specific phylogenetic distances separating classes of taxa. Sequences from hyperthermophiles have a high $\mathrm{G}+\mathrm{C}$ content, which stabilizes the secondary structure at high temperatures. This results in a lower rate of base substitution than that observed for mesophilic organisms such as extreme halophiles and methanogens. While the $\mathrm{G}+\mathrm{C}$ content shows significant variation from one sequence to another, the purine (and therefore, pyrimidine) content of the sequences is nearly constant (Table. 1). Thus, we performed transversion distance analysis to correct the branching order artifacts produced by $\mathrm{G}+\mathrm{C}$ content disparities.

Despite the clustering of the genus Aeropyrum and the genera in the families Pyrodictiaceae and Desulfurococcaceae, the genus Aeropyrum should not be assigned to the family Desulfurococcaceae, since (i) the transversion distance between Aeropyrum pernix and its neighbor Pyrodictium occultum (3.1 substitutions per 100 nucleotides) is as high as that between P. occultum and Desulfurococcus mobilis (3.1 substitutions), each of which belongs to different archaeal families, and (ii) the lack of a common physiological feature is a strong argument that the genus Aeropyrum belongs to neither the family Pyrodictiaceae nor Desulfurococcaceae. Growth of $A$. pernix is not observed under anaerobic conditions in the presence or absence of elemental sulfur, whereas all the members of the families Pyrodictiaceae and Desulfurococcaceae are unable to thrive aerobically. ${ }^{22-26)}$

The aerobic marine hyperthermophile $A$. pernix was 
originally isolated at a single site in Japan but has since been found in several different geographical locations (Nomura et al., Unpublished data). The genus Aeropyrum and its related organisms yet to be discovered might be widespread in the marine hydrothermal environments. Considering that in situ detection of the 16S rDNA fragment revealed the high abundance of marine planktonic crenarchaea in the ocean, ${ }^{27-30)}$ this speculation should not be surprising. The establishment of the new family "Aeropyraceae" might be led after the isolation and detailed characterization of as-yet-undiscovered organisms closely related to the the genus Aeropyrum.

Acknowledgments This work was supported in part by a Grant-in-Aid for Scientific Research (no. 07556048) from the Ministry of Education, Science, Sports and Culture of Japan. N. Nomura is a recipient of a Research Fellowship (no. 9037) of the Japan Society for the Promotion of Science for Young Scientists.

\section{References}

1) K. O. Stetter: Hyperthermophilic procaryotes. FEMS Microbiol. Rev., 18, 149-158 (1996).

2) N. Nomura and Y. Sako: Growth characteristics of hyperthermophiles. Seibutsu-kogaku Kaishi, 76, 259-263 (1998) (in Japanese).

3) Y. Sako, N. Nomura, A. Uchida, Y. Ishida, H. Morii, Y. Koga, T. Hoaki, and T. Maruyama: Aeropyrum pernix gen. nov., sp. nov., a novel aerobic hyperthermophilic archaeon growing at temperatures up to $100^{\circ}$ C. Int. J. Syst. Bacteriol., 46, 1070-1077 (1996).

4) Y. Sako and N. Nomura: Genus Aeropyrum Sako, Nomura, Uchida, Ishida, Morii, Koga, Hoaki, and Maruyama 1996, 1075 in "Bergey's Manual of Systematic Bacteriol, 2nd. Ed., Vol. 1", (ed. by G. M. Garrity et al.), Williams \& Wilkins Co., Baltimore, in press.

5) Y. Sako, P. Chavez Croocker, and Y. Ishida: An extremely heat-stable extracellular proteinase (aeropyrolysin) from the hyperthermophilic archaeon Aeropyrum pernix K1. FEBS Lett., 415, 329 334 (1997).

6) P. Chavez Croocker, Y. Sako, and A. Uchida: Purification and characterization of an intracellular heat-stable proteinase from the marine hyperthermophilic archaeon Aeropyrum pernix K1. Extremophiles, 3, 3-9 (1999).

7) N. Nomura, Y. Sako, and A. Uchida: Molecular characterization and postsplicing fate of three introns within the single rRNA operon of the hyperthermophilic archaeon Aeropyrum pernix K1.J. Bacteriol., 180, 3635-3643 (1998).

8) S. Burggraf, H. Huber, and K. O. Stetter: Reclassification of the crenarchaeal orders and families in accordance with 16S IRNA sequence data. Int. J. Syst. Bacteriol., 47, 657-660 (1997).

9) P. De Rijk, Y. Van de Peer, I. Van den Broeck, and R. De Wachter: Evolution according to large ribosomal subunit RNA. $J$. Mol. Evol, 41, 366-375 (1995).

10) H. Leffers, J. Kjems, L. Ostergaard, N. Larsen, and R. A. Garrett: Evolutionary relationships amongst archaebacteria: A comparative study of $23 \mathrm{~S}$ ribosomal RNAs of a sulfur-dependent extreme thermophile, an extreme halophile and a thermophilic methanogen. $J$. Mol. Biol., 195, 43-61 (1987).

11) L. Achenbach and C. R. Woese: $16 \mathrm{~S}$ and $23 \mathrm{~S}$ rRNA-like primers, in "Archaea, a laboratory manual, thermophiles" (ed. by F. T. Robb and A. R. Place), Cold Spring Harbor Laboratory Press, Cold Spring Harbor, N. Y., 1995, pp. 201-203.
12) C. Yanisch-Perron, J. Vieira, and J. Messing: Improved M13 phage cloning vectors and host strains: nucleotide sequences of the M13 mp18 and pUC19 vectors. Gene, 33, 103-119 (1985).

13) F. Sanger, S. Nicklen, and A. R. Coulson: DNA sequencing with chain-terminating inhibitors. Proc. Natl. Acad. Sci. USA, 74, 5463-5467 (1977).

14) J. D. Thompson, D. G. Higgins, and T. J. Gibson: Clustal w: improving the sensitivity of progressive multiple sequence alignment through sequence weighting, position-specific gap penalties and weight matrix choice. Nucleic Acids Res., 22, 4673-4680 (1994).

15) P. De Rijk, Y. Van de Peer, and R. De Wachter: Database on the structure of large ribosomal subunit RNA. Nucleic Acids Res., 24, 117-123 (1997).

16) R. R. Gutell, N. Larsen, and C. R. Woese: Lessons from an evolving rRNA: 16S and 23S rRNA structures from a comparative perspective. Microbiol. Rev., 58, 10-26 (1994).

17) M. Kimura: A simple method for estimating evolutionary rates of base substitutions through comparative studies of nucleotide sequences. J. Mol. Evol., 16, 111-120 (1980).

18) N. Saitou and M. Nei: The neighbor-joining method: a new method for reconstructing phylogenetic trees. Mol. Biol. Evol., 4, 406-425 (1987).

19) C. R. Woese, L. Achenbach, P. Rouviere, and L. Mandelco: Archacal phylogeny: Reexamination of the phylogenetic position of Archaeoglobus fulgidus in light of certain composition-induced artifacts. Syst. Appl. Microbiol, 14, 364-371 (1991).

20) J. Felsenstein: Confidence limits on phylogenies: an approach using bootstrap. Evolution, 39, 783-791 (1985).

21) G. J. Olsen, C. R. Woese, and R. Overbeek: The wind of (evolutionary) change: Breathing new life into microbiology. J. Bacteriol., 176, 1-6 (1994).

22) W. Zillig, K. O. Stetter, D. Prangishvilli, W. Schafer, S. Wunderl, D. Janekovic, I. Holz, and P. Palm: Desulfuroccoccaceae, the second family of the extremely thermophilic, anaerobic, sulfur-respiring Thermoproteales. Zentrabl. Bakteriol. Mikrobiol. Hyg. 1. Abt. Org. C, 3, 304-317 (1982).

23) K. O. Stetter, H. Konig, and E. Stackebrandt: Pyrodictium gen. nov., a new genus of submarine disc-shaped sulphur reducing archaebacteria growing optimally at $105^{\circ} \mathrm{C}$. Syst. Appl. Microbiol., 4, 535-551 (1983).

24) U. Pley, J. Schipka, A. Gambacorta, H. W. Jannasch, H. Fricke, R. Rachel, and K. O. Stetter: Pyrodictium abyssi sp. nov. represent a novel heterotrophic marine archaeal hyperthemophile growing at $110^{\circ} \mathrm{C}$. Syst. Appl. Microbiol., 14, 245-253 (1991).

25) G. Fiala, K. O. Stetter, H. W. Jannasch, T. A. Langworthy, and J. Madon: Staphylothermus marinus sp. nov. represents a novel genus of extremely thermophilic submarine heterotrophic archaebacteria growing up to $98^{\circ} \mathrm{C}$. Syst. Appl. Microbiol., 8, 106-113 (1986).

26) W. Zillig, I. Holz, D. Janekovic, H. - P. Klenk, E. Imsel, J. Trent, S. Wunderl, V. H. Forjaz, R. Coutinho, and T. Ferreira: Hyperthermus butylicus, a hyperthermophilic sulfur-reducing archaebacterium that ferments peptides. J. Bacteriol., 172, 3959-3965 (1990).

27) E. F. Delong: Novel archaea in coastal marine environments. Proc. Natl. Acad. Sci. USA, 89, $5685-5689$ (1992).

28) E. F. DeLong, K. Y. Wu, B. B. Prezelin, and R. V. M. Jovine: High abundance of Archaea in Antarctic marine picoplankton. Nature, 371, 695-697 (1994).

29) J. A. Fuhrman, K. McCallum, and A. A. Davis: Novel major archaebacterial group from marine plankton. Nature, 356, 148-149.

30) J. A. Fuhrman, K. McCallum, and A. A. Davis: Phylogenetic diversity of subsurface marine microbial communities from the Atlantic and Pacific Oceans. Appl. Environ. Microbiol., 59, 1294-1302 (1993). 